\title{
The Development of a Questionnaire Survey to Investigate the Critical Risk Factors in Oil and Gas Pipelines Projects
}

\author{
Layth Kraidi a,b*, Raj Shah ${ }^{\text {a }}$, Wilfred Matipa a and Fiona Borthwick ${ }^{\text {a }}$ \\ ${ }^{a}$ Department of the Built Environment, Faculty of Engineering and Technology, Liverpool John Moores University. \\ Liverpool, L3 3AF, England, UK \\ ${ }^{\mathrm{b}}$ Department of Civil Engineering, College of Engineering, Al Muthanna University, \\ Al Muthanna, Iraq
}

\begin{abstract}
The scarcity of data about "the probability and severity" of the Risk Factors (RFs) and "the usability and effectiveness" of the Risk Mitigation Methods (RMMs) in Oil and Gas Pipelines (OGPs) are hindering the efforts of risk mitigations in these projects. Consequently, this paper aims to develop a questionnaire survey to collect these require data to analyze the RFs and effectively evaluate the RMMs. Firstly, documents qualitative analysis were carried out to identify the RFs and RMMs in OGPs projects in different countries worldwide. Secondly, an industry-wide questionnaire survey was found to be an effective quantitative approach to analyze the "probability and severity" levels of the RFs and to evaluate the "usability and effectiveness" degrees of the RMMs. A pilot-like survey was significantly needed to improve the clarity of the questions and revise the ambiguous questions. As well as, to add the necessary queries and discard the unnecessary ones. Moreover, the pilot-like survey was used to test the functionality of the rating scales; and to improve the overall design of the survey. This survey filled by a number of experts in OGPs projects; their feedback was found helpful to write the final draft of the survey. The findings of this paper was a questionnaire survey that will be used in ongoing research about mitigating the RFs in OGPs projects. Furthermore, a few authors explained their procedure of designing such survey. Therefore, researchers in this field could use the findings and comments of this to design their surveys.
\end{abstract}

Keywords: Oil and gas pipelines (OGPs), risk analysis, Risk Mitigation Methods (RMMs), questionnaire survey, pilot-like survey, stakeholders perceptions

\section{Introduction}

The procedure of risk management in Oil and Gas pipelines (OGPs) projects requires a proper knowledge [1] and verified historical records [2] about the probability and severity levels of the Risk Factors (RFs) that associated with OGPs projects. However, the current methods of RFs analysis are not accurate enough to analyze the probability and severity levels of the RFs. Particularly, in the developed and troubled countries because no database provides verified information about the RFs has been established yet [3,4]. Moreover, up-to-date data about the "usability and effectiveness" degrees of Risk Mitigation Methods (RMMs) are required to manage the safety of OGPs more effectively and to make useful recommendations for OGP's risk management. From the preceding, there is a vital need to collect trusted data about the "probability and severity levels" of RFs and about "the usability and effectiveness degrees" of RMMs.

Therefore, this paper aims to design a questionnaire survey to collect the required data for mitigating the RFs in OGPs projects such as (I) identify the RFs; (II) analyze the portability and severity of the RFs; (III) identify the RMMs and (IV) evaluate the usability and effectiveness degree of the RMMs.

Moving forward in this paper, section 2 was about identifying RFs and RMMs in OGPs projects. Section 3 illuminates the procedure of developing a questionnaire survey. Section 4 shows the final draft of the questionnaire survey. Section 5 discusses the findings of this paper. Finally, section 6 highlights the conclusions. 


\section{Identifying the RFs and RMMs}

Worldwide qualitative documents analysis were carried out to identify the RFs and RMMs in OGPs projects in different countries. Extra attention was made to identify the RFs in the insecure areas, the findings of these investigations are shown in Table 1.

Table 1. The identified RFs and RMMs in OGPs projects from the documents analysis.

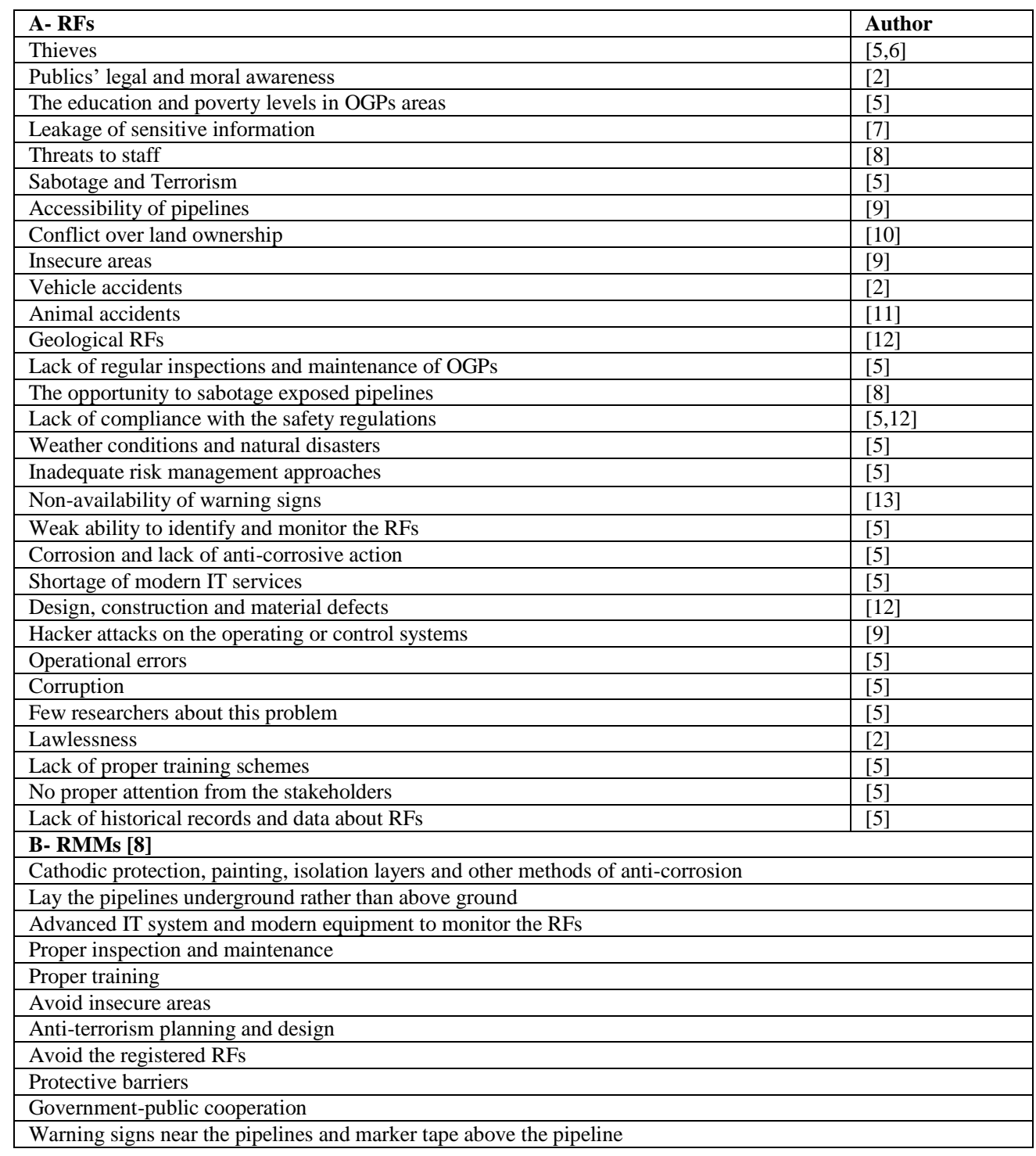

However, the results of the investigations in Table 1 cannot provide information about the "probability and severity" levels of the RFs and the "usability and effectiveness" degrees of the RMMs in a specific country. Especially, in the countries which have limited registrations abut RFs and limited studies about the safety of OGPs such as Iraq. Therefore, this paper was aimed to design a questionnaire survey to engage with the stakeholder in OGPs projects to obtain consensus perceptions about that RFs and RMMs in OGPs projects. Because the perceptions of stakeholders are based on real experience in OGPs projects, which makes them qualified to monitor the RFs in OGPs projects [14] and to evaluate the RMMs. 


\section{Structure Development of the Questionnaire Survey}

Questionnaire surveys are one of the most widely used data collection methods to understand an attitude or behavior. As well as, this method of data collection enables the researchers to write the formulation of precise queries for respondents whose views are needed [15]. For an accurate questionnaire survey, the design of the survey went through different steps; the flowchart of developing the questionnaire survey was illuminated in Figure 1.

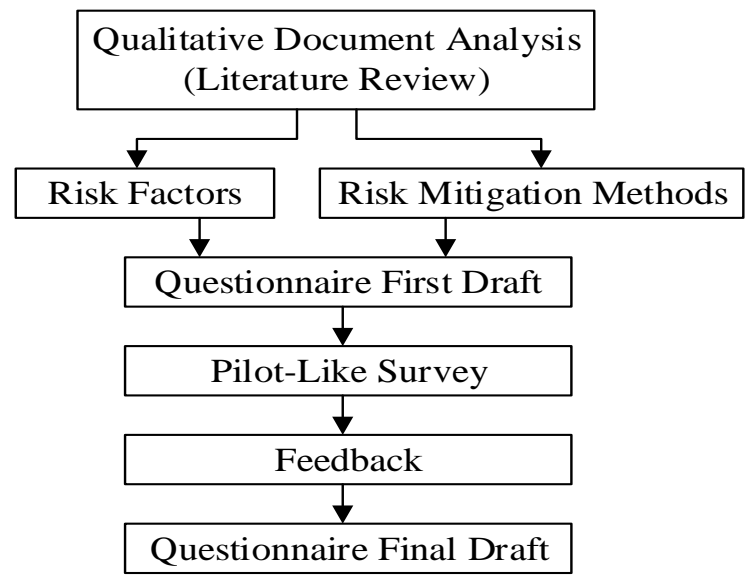

Figure 1: The flowchart of developing the questionnaire survey.

An extensive review of the literature was accomplished to determine the variables of the survey questionnaire that are the RFs and RMMs (see Table 1). Firstly, Table 1 was adopted to write the first draft of the questionnaire (see Table 2). Secondly, send this draft to a number of experts in OGPs projects in Iraq to get their feedback about the survey. The final step is to use work with the experts' feedback to write the final draft of the survey. 
Table 2: The first draft of the questionnaire survey.

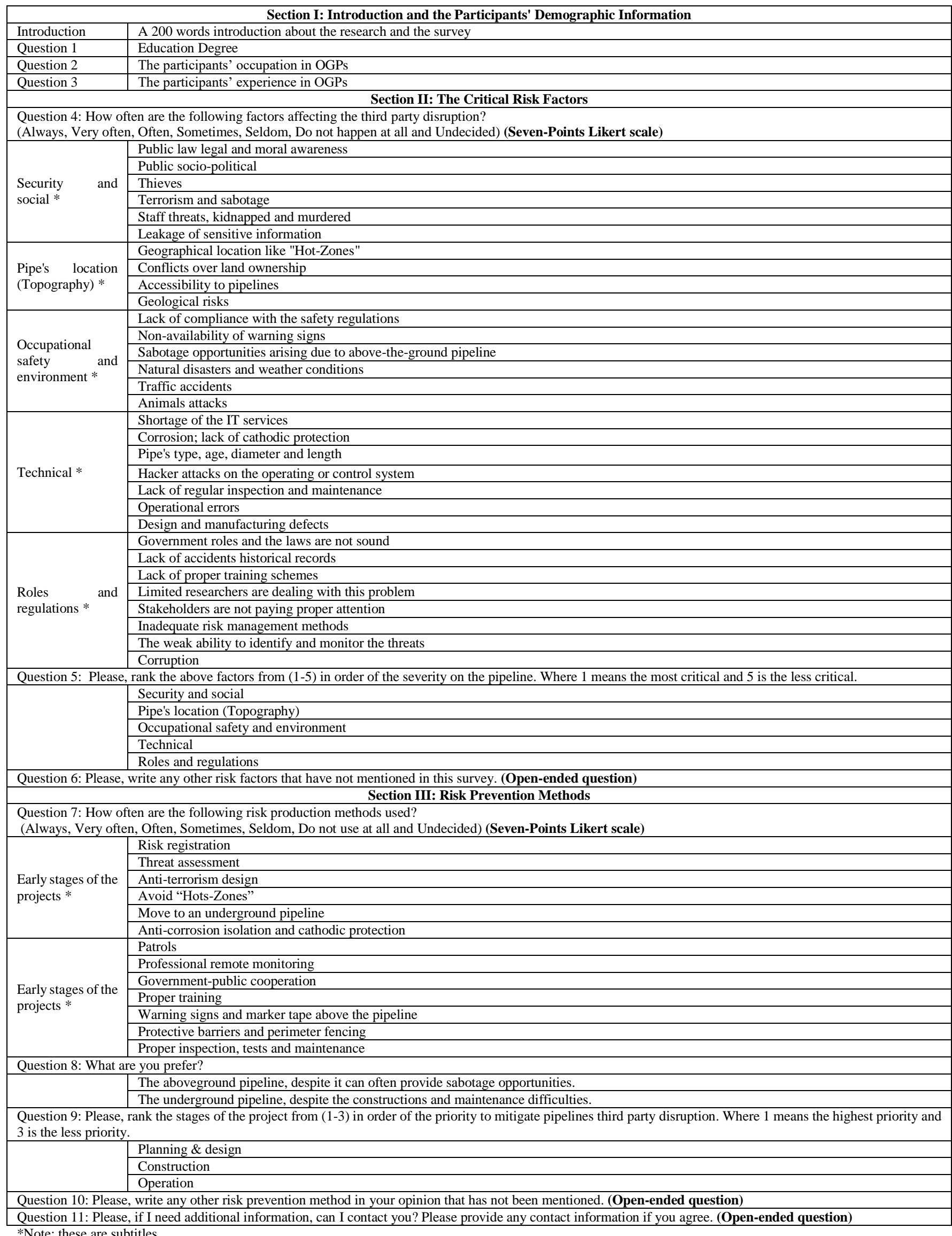

*Note: these are subtitles. 
The first draft of the survey had three sections as follows. Section I was about a brief summary about the research to explain the aim of this research, the purpose of the survey, a notification that the respondents will be treated anonymously, and the research's contact details "email and the mobile number" in case of any inquiries. Questions 1 to 3 were asked about the participants' degree of education, occupation and experience respectively. Section II had three questions to evaluate the RFs and to add more RFs for the study. Finally, section III had five questions to evaluate the RMMs and to add more RMMs for the study.

A pilot-like test refers to a pre-test that estimates the response rate of the targeted sample. The purpose of a pilot-like is to spot certain aspects of the survey that needs refinement. Also, it helps the researcher to predict the factors that might affect the validity of the survey to avoid them [16]. Over and above, the first draft of the survey was adopted in a pilot survey to assess the clarity of the questions, the functionality of the rating scales and about the overall consistency and design of the questionnaire. The survey was written in English and Arabic languages, and it was up to the respondents to choose the language. As Blaxter et al. [15] recommended, the pilot survey was sent to 10 experts in OGPs projects in Iraq for an informal discussion about the survey. After one week, six of these experts filled the pilot survey as shown in Table 3.

Table 3: Experts' general information.

\begin{tabular}{|l|l|l|l|l|l|}
\hline \multicolumn{2}{|c|}{ Education } & \multicolumn{2}{c|}{ Experience } & \multicolumn{2}{c|}{ Experience (years) } \\
\hline No degree & 2 & a member of a construction team & 5 & 6 to 10 & 1 \\
\hline Bachelor degree or Higher diploma & 2 & a researcher or student & 1 & 11 to 15 & 2 \\
\hline Masters or PhD & 2 & & & More than 15 & 2 \\
\hline Total & 6 & Total & 6 & Total & 6 \\
\hline
\end{tabular}

Working with comments from experts was as follows. The subtitles are making the survey long. Therefore, the subtitles have been removed from the final survey. The pilot-like survey missed evaluating the severity levels of the RFs and the effectiveness degrees of the RMMs. For that purpose, questions 5 "to analyze the severity of the RFs" and 9 to "evaluate the effeteness of the RMMs" were added in the final survey. Likert scale was used in this survey because it one of the most and widely used scales for despite critiques like previous studies were reported the respondents might be biased or attempt to portray the issues in a more personal matter [17-19]. The authors wanted to analyze the RFs and evaluate the RMMs more effectively by using a seven-point Likert scale. However, the participants complained that it was confusing to them. Thus, a five-point Likert scale was used in the final survey, which is more comfortable for follow as they suggested. After phone calls with the participants, the clarity of the survey overall was improved, the questions that found to be vague were revised or discarded, the lists of RFs and RMMs methods were revised for better clarity. Some of these RFs and RMMs have been paraphrased to make sure that these lists fit with the aim of the ongoing research about mitigating the RFs in OGPs projects in Iraq. Some typos, spellings and grammar mistakes were spotted in the pilot survey and changed in the final draft. The statistical analysis of the pilot like survey were used to test the functionality of the survey for the research. From the preceding, the final draft of the survey was written as explained in section 4.

\section{The Final Draft of the Questionnaire Survey}

The final draft of the questionnaire survey was shown in Table 4.

Table 4: The final draft of the questionnaire survey.

$$
\text { Section I: Introduction and the Participants' Demographic Information }
$$

\begin{tabular}{|l|l|}
\hline \multicolumn{2}{|c|}{ Section I: Introduction and the Participants' Demographic Information } \\
\hline Introduction & A 200 words introduction about the research and the survey \\
\hline Question 1 & Education Degree \\
\hline Question 2 & The participants' occupation in OGP projects \\
\hline Question 3 & The participants' experience in OGPs projects \\
\hline
\end{tabular}




\section{Section II: Analyzing the Risk Factors}

Please, rank the following risk factors which are facing the oil and gas pipeline projects on the scale of probability and severity. Please note, to see the two scales, You may need to move the screen to the right or the left.

Question 4: Risk factors probability scale. (Almost certain, Likely, Possible, Unlikely and Rare) (Five-Points Likert scale)

Question 5: Risk factors severity and consequence scale. (Catastrophic, Major, Moderate, Minor and Negligible) (Five-Points Likert scale)

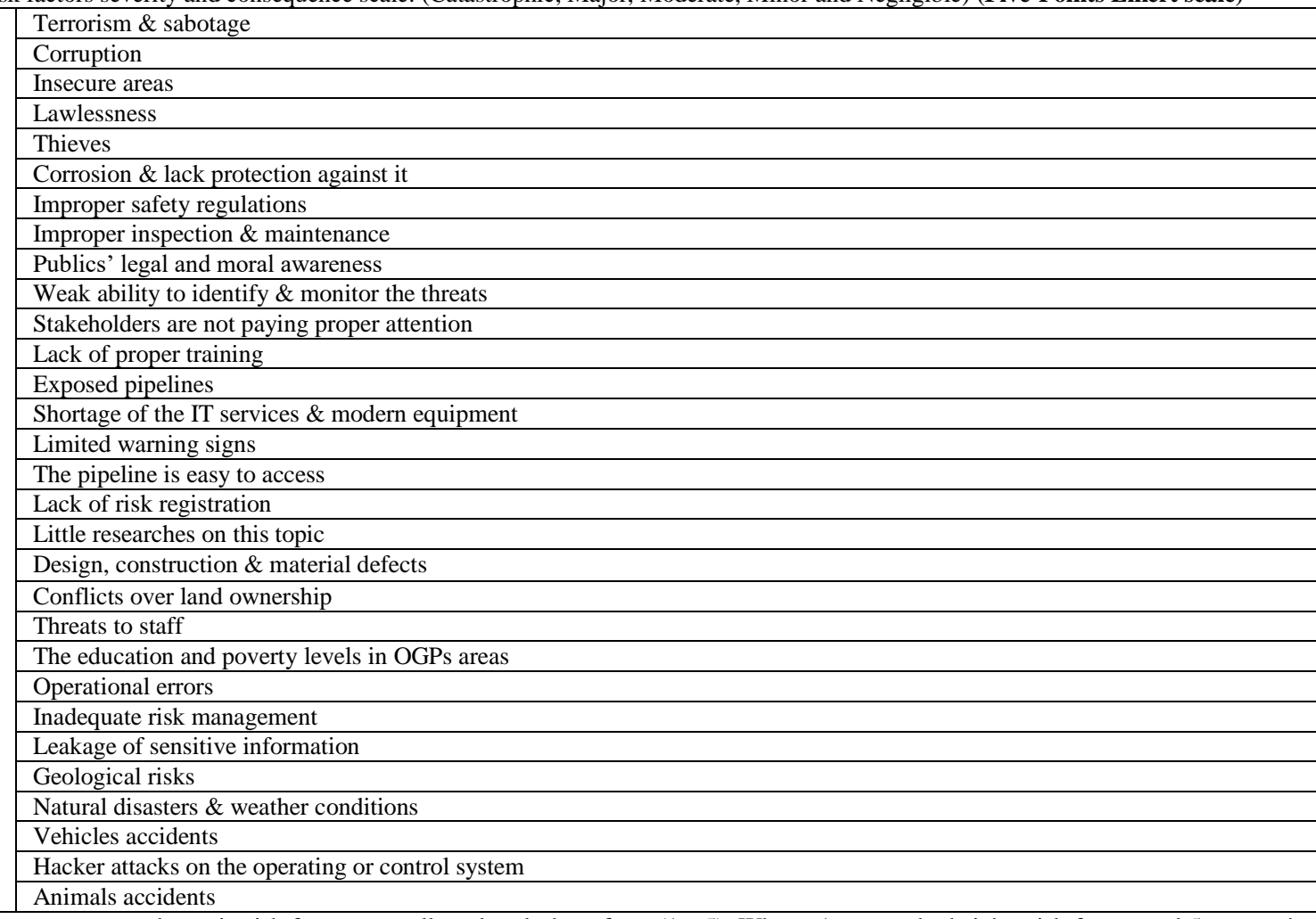

Question 6: Please, compare the main risk factors overall, and rank them from (1 - 5). Where: 1 means the heights risk factor, and 5 means the lowest risk.
Security \& Social (S\&S)
Pipes' Location (PL)
Health, Safety and Environments (HSE)
Rules and Regulation (R\&R)
Operational Constraints (OC)

Question 7: Please, write any other risk factor in your opinion that has not been mentioned

\section{Section III: Evaluating Risk Mitigations Methods}

Please, rank the following risk protection methods regarding the degree of applications and effectiveness.

Question 8: Protection methods usage scale. (Almost certain used, Likely used, Possible used, Unlikely used and Rare used) (Five-Points Likert scale)

Question 9: Protection methods effectiveness scale. (Extremely effective, Very effective, Moderately effective, Slightly effective and Ineffective) (Five-Points Likert scale)

\begin{tabular}{|l|}
\hline Avoid "Insecure-Zones \\
\hline Anti-terrorism design \\
\hline Avoid the registered risks and threats \\
\hline Proper training \\
\hline Move to an underground pipeline \\
\hline Anti-corrosion such as isolation and cathodic protection \\
\hline Protective barriers and perimeter fencing \\
\hline Warning signs and marker tape above the pipeline \\
\hline Foot and vehicles patrols \\
\hline High technology and professional remote monitoring \\
\hline Government-public cooperation \\
\hline Proper inspection, tests and maintenance \\
\hline Question 10 Projects' stages \\
\hline Planning \& design stage \\
\hline Construction stage
\end{tabular}




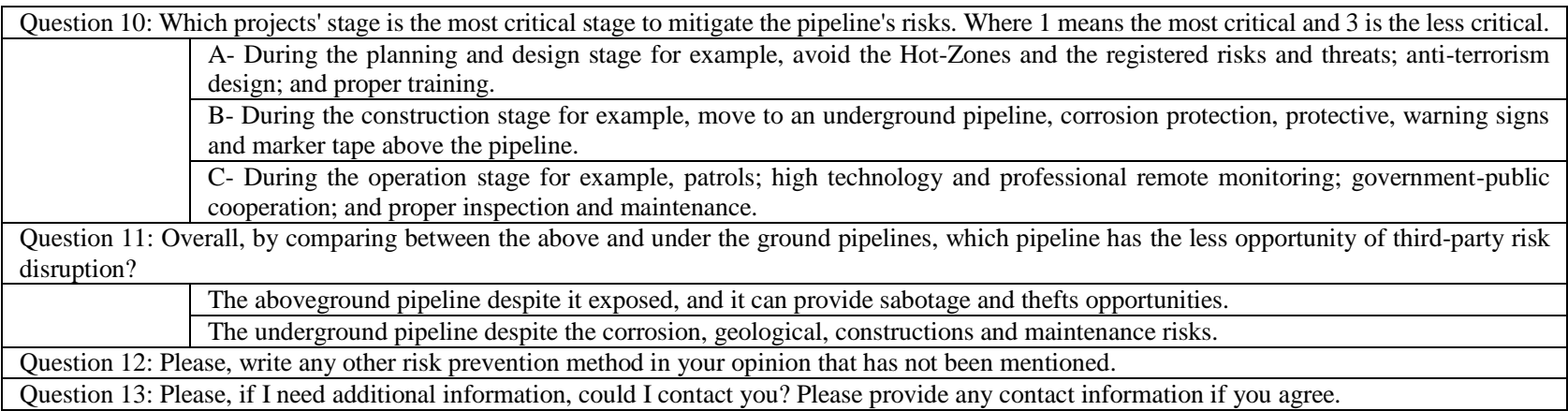

The final draft of the survey had 13 questions divided in three sections as follows. Similar to the pilot-like survey, section I explained an introduction to the survey. The first three questions were asked about the participants' occupation, experience, and degree of education. Section II of the questionnaire survey comprised four questions to analyze the RFs. Question 4 asks about the probability levels of the RFs. Question 5 asks about the severity levels of the RFs. Question 6 asks to rank the RFs by their degree on influence on OGPs. And, question 7 was an open-ended question to add more RFs for the survey by the participants. Section III: had five questions to evaluate the RMMs. Question 8 askes to evaluate the usability of the RMMs. Question 9 askes to evaluate the effectiveness of the RMMs. Question 10 asks to rank the stages of pipelines' projects regarding the priority of mitigating the RFs. Question 11 was about an overall comparison between aboveground and underground pipeline with the subject to RFs. The final question was to collect the participants' contact details.

\section{Discussion}

Questionnaire surveys have always been used as data collection methods in social researchers and operations strategy researchers. A well-structured questionnaire survey and clear and to the point questions considered proper because, they made the survey easier for the respondents and also allowed the researcher focus on the variables of the survey, which makes the analysis of the survey easier to them. Moreover, it is essential to be aware of the ethical considerations in the survey to protect the privacy of the participants.

Sampling means to select some from a larger group to estimate or generalize the dominance of an unknown chunk of information [20]. The snowball sampling technique was applied for this survey to ensure widespread distribution of the survey [21,22]. This technique works as follow; the survey will initially distribute to some previously identified participants who will be asked to forward it to others until the required number of responses is reached [21].

An online tool was chosen to distribute the survey because it is a quick method of data collection compared to mail and paper survey, easy to manage, less cost and environment friendly [23]As well as, the online survey provides a chance to the participants to cooperate and explain their idea about the via open-ended questions [24]. However, this kind of survey might have a low response rate that results from some disadvantages like computer and website literacy, the targeted population or some of them might not have access to the Internet and web security issues [25].

\section{Conclusion}

This paper describes an aspect of developing a questionnaire survey as part of ongoing research about mitigating the RFs in OGPs projects. The survey will use an online tool to recruit respondents that have relevant experience with OGPs projects such as planners, designers, consultants, construction workers and operators.

Collecting the required data by using questionnaire surveys could reduce the time and cost of investigations, increase the stakeholders' awareness about their responsibilities regarding OGPs risk management. However, it depends on stakeholders' willingness to cooperate with the authors, which is one of this method's main advantages. Collecting the required information from various and trusted sources such as previous studies and stakeholders could provide additional understanding and knowledge about OGPs' safety. Also, the collected data could provide reliable and valid data about 
mitigating RFs in OGPs projects to analyze the RFs more accurately. Moreover, it helps to identify the positive and negative recommendations about RMMs in a way that ensure the planes and strategies for pipelines' safety.

\section{Acknowledgement}

The support of the financial sponsor "The Ministry of Higher Education and Scientific Research in Iraq" is highly appreciated by the authors.

\section{References}

[1] B. Anifowose, D. M. Lawler, D. Van Der Horst, L. Chapman. Attacks on oil transport pipelines in Nigeria: A quantitative exploration and possible explanation of observed patterns. Applied Geography. 32 (2012) 636-651.

[2] D. Prochazkova. Critical Infrastructure and Region Safety. Models and Methods in 332 Applied Sciences. (2010) 47-52.

[3] X. Y. Peng, D. C. Yao, G. C. Liang, J. S.Yu, S. He, S. Overall reliability analysis on oil/gas pipeline under typical third-party actions based on fragility theory. Journal of Natural Gas Science and Engineering. 34 (2016) 993-1003.

[4] D. Ge, M. Lin, Y. Yang, R. Zhang, Q. Chou. Reliability analysis of complex dynamic fault trees based on an adapted K.D. Heidtmann algorithm. Proceedings of the Institution of Mechanical Engineers, Part O: Journal of Risk and Reliability. 229(6) (2015) 576-586.

[5] U. Nnadi, Z. El-Hassan, D. Smyth, J. Mooney. Lack of proper safety management systems in Nigeria oil and gas pipelines. IChemE Institution of Chemical Engineers. 237, (2014) 27- 34.

[6] F. C. Onuoha. Oil pipeline sabotage in Nigeria: Dimensions, actors and implications for national security. African Security Review 17 (2008) $99-$ 115.

[7] W. S. Wu, C. F. Yang, J. C. Chang, P. A. Château, Y. C. Chang. Risk assessment by integrating interpretive structural modeling and Bayesian network, case of offshore pipeline project. Reliability Engineering \& System Safety. 142 (2015) 515-524.

[8] A. Rowland. GIS-based prediction of pipeline third-party interference using hybrid multivariate statistical analysis. PhD, Newcastle University, 2011.

[9] A. Srivastava \& J. Gupta, New methodologies for security risk assessment of oil and gas industry, J. process safety and environmental protection, 88(6), (2010) 407-412.

[10] k. Macdonald \& A. Cosham, Best practice for the assessment of defects in pipelines-gouges and dents, J. Engineering Failure Analysis, 12(5), (2005) 720-745.

[11] S. Mubin \& G. Mubin, Risk analysis for construction and operation of gas pipeline projects in Pakistan. Pakistan Journal of Engineering and Applied Sciences, 2(Jan) 2008, 22-37.

[12] Y. Guo, X. Meng, D. Wang, T. Meng, S. Liu \& R. He, Comprehensive risk evaluation of long-distance oil and gas transportation pipelines using a fuzzy Petri net model, J. of Natural Gas Science and Engineering, 33 (2016) 18-29.

[13] U. Nnadi, Z. El-Hassan, D. Smyth, \& J. Mooney, Lack of proper safety management systems in Nigeria oil and gas pipelines. Delta, 2007, 13th. IChemE, SYMPOSIUM SERIES NO 159, HAZARDS 24, 1999.

[14] E. Sa'idi, B. Anvaripour, F. Jaderi, N. Nabhani. Fuzzy risk modeling of process operations the oil and gas refineries. Journal of Loss Prevention in the Process Industries. 30 (2014) 407 63-73.

[15] L. Blaxter, C. Hughes, M. Tight M, How to Research, 4th ed. Berkshire, England: McGraw Hill Education, 2010.

[16] L. Neuman, Social Research Methods-Qualitative and Quantitative approaches. 7th ed. New York: Pearson Education InC, 2011.

[17] K. Mearns and S. Yule, 'The role of national culture in determining safety performance: Challenges for the global oil and gas industry', Safety Science, 47, (6), (2009) pp. 777-785.

[18] R. A Kent, Data construction and data analysis for survey research, Palgrave MacMillan, 2011.

[19] M. S. Matell and J. Jacoby, (1971) 'Is there an optimal number of alternatives for Likert scale items? Study I: Reliability and validity', 31, (3), pp. 657.

[20] R. Kumar, Research Methodology. 1st ed. London: Sage Publications Limited, 2005.

[21] R., F., M. Ameen, M. Mourshed. Environmental, Social and Economic Challenges for Urban Development: Stakeholder's Perception in a Developing Economy. Proceedings of the International Conference on Computing in Civil and Building Engineering 2016. Osaka, Japan. ISBN: 9784-9907371-2-2, 2016.

[22] I. M. Dragan, A. Isaic-Maniu. Snowball sampling completion. Journal of Studies in Social Sciences. 5 (2013) 160-177.

[23] S. Dolnicar, C. Laesser, K. Matus. Online versus paper: format effects in tourism surveys. Journal of Travel Research. Vol. 47, issue 3, pp. 295-

316. 2009.

[24] J. C. Bertot. Web-Based Surveys: Not Your Basic Survey Anymore. The University of Chicago Press Journal. Vol. 79, No. 1, pp. 119-124. 2009.

[25] J. Blair, R. Czaja. J. Blair. Designing surveys: A guide to decisions and procedures, Pine Forge Press. SAGE. London. ISBN 978-1-4129-97348. 2013. 